\title{
Comparación multitemporal de áreas quemadas en un bosque seco tropical utilizando el índice de área quemada (IAQ)
}

\author{
Multitemporal comparison of burned areas in a tropical dry forest using the burned area \\ index (BAI)
}

\author{
Daniela Vargas-Sanabria ${ }^{1}$ (D) $\bullet$ Carlos Campos-Vargas
}

Recibido: 07/02/2020 Aceptado: 02/04/2020 Publicado: 28/07/2020

\begin{abstract}
Post-fire analysis such as the burned area index (BAl) allows us to discriminate affected areas and the severity of fires. In this study, the annual behaviour of the BAI was analyzed in the tropical dry forest region of the Guanacaste Conservation Area, Costa Rica among 1997 to 2019 according to the frequency of fires. Our results show that the areas frequently burned by forest fires display a differentiated behaviour in the BAI throughout the years. Where, the greater the frequency of forest fires is, the higher the BAI values. On the other hand, areas that have not been affected or have been affected by a single fire showed low BAI values. These results could validate the hypothesis that after two to three fires, the forest seed bank is destroyed. Consequently, the composition and structure of tropical dry forests change considerably after the occurrence of many fires.
\end{abstract}

Key words: Spectral index, wildfires, remote sensing, fire frequency.

1. Laboratorio de Investigación e Innovación Tecnológica, Universidad Estatal a Distancia. San José, Costa Rica.

dvargass@uned.ac.cr; ccamposv@uned.ac.cr 


\section{Resumen}

Los análisis post incendio, como el índice de área quemada (IAQ), permiten discriminar áreas afectadas y severidad de los incendios forestales. En este estudio se comparó según la frecuencia de incendios, el comportamiento anual del IAQ en la región de bosque seco tropical del Área de Conservación Guanacaste, Costa Rica entre los años 1997 al 2019. Los resultados evidencian que las áreas frecuentemente quemadas por incendios forestales muestran un comportamiento diferenciado en el IAQ a lo largo de todos los años. Mostrando una relación directa entre la frecuencia de incendios forestales y los valores de IAQ. Donde a mayor frecuencia de incendios forestales, los valores de IAQ son mayores. Por otro lado, las áreas que han sido poco afectadas o no han sido afectadas del todo mostraron valores bajos de IAQ. Estos resultados podrían validar la hipótesis de que después de dos a tres incendios, el banco de semillas es completamente destruido. Esto debido a que la composición y estructura de los bosques secos tropicales cambia considerablemente después de múltiples incendios.

Palabras clave: Índice espectral, incendio forestal, teledetección, frecuencia de incendios.

\section{Introduction}

En bosques secos tropicales, hay una alta probabilidad de que los incendios forestales se conviertan en la perturbación más dominante, debido a la rápida expansión de las fronteras agrícolas, los cambios en la cubierta terrestre y las frecuentes sequías resultado del cambio climático [1], [2]. En Centroamérica, la mayoría de los incendios forestales en los bosques secos están relacionados con quemas provocadas para la preparación de campos agrícolas y limpieza de terrenos [3].

Los impactos de los incendios sobre la biodiversidad y las reservas de carbono están relacionados con la frecuencia e intensidad de los incendios [4]. El fuego tiene el potencial de transformar grandes áreas de bosque seco tropical en ecosistemas de arbustos o sábanas, creando así una retroalimentación positiva en la futura susceptibilidad al fuego, exacerbando la carga de combustible y la intensidad del fuego [1]. Las áreas afectadas se vuelven más susceptibles a los incendios recurrentes a medida que disminuye la cobertura del dosel, disminuye la humedad del sotobosque y la carga de combustible aumenta [5].
Mediante la teledetección se ha logrado detectar perturbaciones, como incendios forestales o deforestación, y se considera el método más fiable para medir y evaluar las métricas de la cubierta vegetal y terrestre a nivel de paisaje [6], [7]. En el análisis de áreas quemadas por medio de teledetección, Chuvieco et al. [8], sugirieron tres líneas de investigación: evaluación de nuevos sensores; desarrollo o adopción de métodos de discriminación de tierra quemada, regresión logística y detección de cambios; y análisis espectral de áreas quemadas para mejorar la exactitud de índices de vegetación.

Los índices espectrales de vegetación son comúnmente usados para determinar propiedades de la vegetación. Estos índices se enfocan en la interpretación de la firma espectral de los objetos que interactúan con la radiación solar [9]. Sin embargo, no todos estos índices fueron diseñadosparadiscriminarsuperficies deáreasquemadas y pueden no ser bien adaptados para diferenciar carbón el cual es el material predominante derivado de áreas afectadas por fuego [10]. El índice de área quemada (IAQ) es un índice de vegetación post fuego que permite identificar las áreas quemadas gracias a la relación de bandas espectrales en las regiones rojo e infrarrojo cercano. Chuvieco et al. [8], encontraron que el IAQ proporcionó la mayor capacidad de discriminación entre otros índices espectrales como Normalized Difference Vegetation Index (NDVI), Soil Adjusted Vegetation Index (SAVI) y el Global Environmental Monitoring Index (GEMI). Por lo tanto, este estudio busca comparar la respuesta espectral del índice de área quemada (IAQ) de forma anual en áreas quemadas del bosque seco tropical del Área de Conservación Guanacaste, Costa Rica, durante el período 1997 al 2019.

\section{Materiales y métodos}

\section{Área de estudio}

El área de estudio abarca seis sectores del Área de Conservación Guanacaste (ACG), Guanacaste, Costa Rica: Santa Rosa, Murciélago, Santa Elena, Junquillal, El Hacha y Pocosol, con una superficie de 57.560 hectáreas. Estos sectores fueron seleccionados debido a su importancia ecológica, ya que, albergan ecosistemas de bosque seco tropical que son afectados de manera recurrente por incendios forestales; además estos sitios han sido monitoreados a lo largo del tiempo como parte de un programa permanente de monitoreo de incendios forestales [11], [12] (Figura. 1).

El área de estudio presenta una temperatura media anual de $25^{\circ} \mathrm{C}$ y dos estaciones, lluviosa y seca, con un promedio de precipitación anual de $1500 \mathrm{~mm}$ [13]. No 
obstante, entre $85 \%$ y el $97 \%$ de las precipitaciones caen entre mayo y noviembre durante la temporada de lluvias; mientras que la estación seca se extiende desde finales de diciembre hasta mediados de mayo con menos de $10 \mathrm{~mm}$ por mes [14].

\section{Colección de datos}

El índice de área quemada (IAQ) está diseñado para enfatizar la señal del carbón en imágenes posteriores al incendio por medio de porciones de la banda roja e infrarrojo cercano (IR) del espectro electromagnético [8]. Este índice se crea siguiendo la ecuación 1.

$$
I A Q=\frac{1}{\left(\operatorname{Red}_{\text {reference }}-\operatorname{Red}_{\text {pixel }}\right)^{2}+\left(N I R_{\text {reference }}-N I R_{\text {pixel }}\right)^{2}}
$$

Donde Red reference $_{\text {y NIR }}$ reference corresponden a los valores de reflectancia de referencia establecidos por Chuvieco et al. [8], cuyo valor fue 0,1 y 0,06 , respectivamente; y

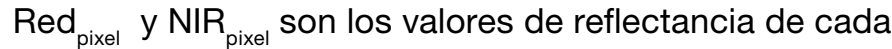
píxel en las mismas bandas. Los datos anuales de IAQ fueron obtenidos por medio de Google Earth Engine (GEE) [15], que es una plataforma web que combina un catálogo de imágenes de satélite y conjuntos de datos geoespaciales que permiten el análisis de gran cantidad de datos. Los mosaicos de IAQ fueron construidos con colecciones de escenas LANDSAT para el período de 1997 al 2019 (Cuadro 1). Dichas colecciones cuentan con una ortorectificación a un nivel de procesamiento estándar L1 - TIER1 y fueron transformadas de radiancia a reflectancia mediante el Algoritmo del Sistema de Procesamiento Adaptativo de Interferencia del Ecosistema de Landsat (LEDAPS, por sus siglas en inglés), así como por el Código de Reflectancia Superficial Landsat (LaSRC, por sus siglas en inglés) en el caso de la colección de Landsat 8.

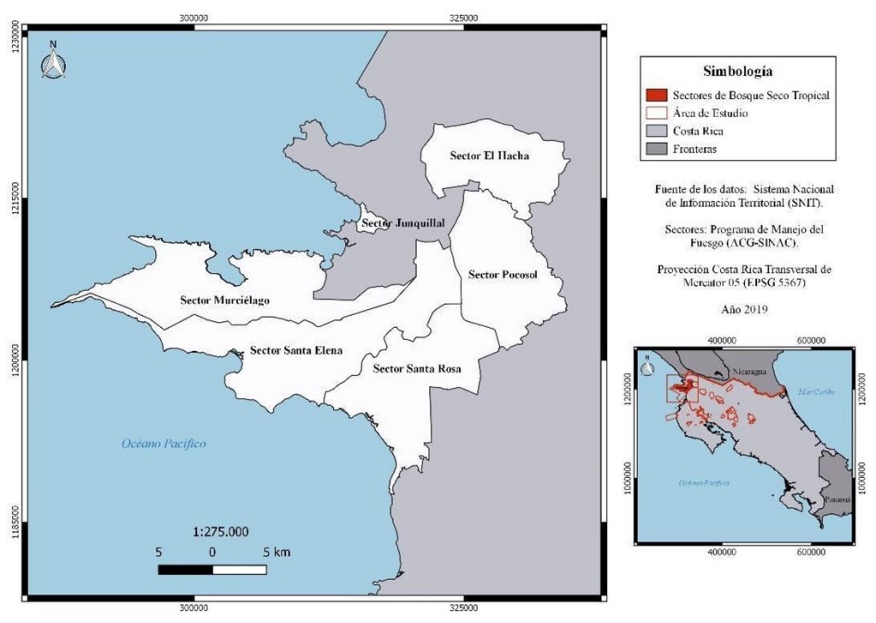

Figura 1. Ubicación del área de estudio en la región del bosque seco del Área de Conservación Guanacaste (ACG), Costa Rica.

Figure 1. Location of the study area in the dry forest region of the Guanacaste Conservation Area (ACG), Costa Rica.

Los polígonos de área quemada, para el período 1997 al 2019, fueron recopilados mediante trabajo de campo por el Programa Manejo del Fuego del Área de Conservación Guanacaste del Sistema Nacional de Áreas de Conservación de Costa Rica. Estos polígonos de incendio fueron recolectados siguiendo el perímetro de las zonas afectadas tan pronto como se extinguieron los incendios, utilizando un equipo GPS con la opción de seguimiento activo y una precisión media de 3 metros. Posteriormente, los polígonos recolectados en campo fueron transformados en formato vectorial y agrupados por temporada de incendio, con el fin de contar con un archivo vectorial para cada temporada de incendios que permitiera conocer el lugar donde ocurrió el incendio, el número de hectáreas afectadas y la fecha en la

Cuadro 1. Características de los mosaicos anuales de índice de área quemada (IAQ) utilizados en la región de bosque seco tropical del ACG, 1997 al 2019.

Table 1. Characteristics of the annual burned area index (BAI) mosaics used in the ACG tropical dry forest region, 1997 to 2019.

\begin{tabular}{|c|c|c|c|}
\hline $\begin{array}{c}\text { Periodo } \\
\text { (Años) }\end{array}$ & Sensor & Transformación a reflectancia & Motor de búsqueda GEE \\
\hline $1997-2000$ & $\begin{array}{c}\text { Landsat } 5 \\
\text { ETM }\end{array}$ & LEDAPS & ee.ImageCollection("LANDSAT/LT05/C01/T1_ANNUAL_BAI \\
\hline $2000-2003$ & $\begin{array}{c}\text { Landsat } 7 \\
\text { ETM+ }\end{array}$ & LEDAPS & ee.ImageCollection("LANDSAT/LE07/C01/T1_ANNUAL_BAI \\
\hline $2003-2012$ & $\begin{array}{c}\text { Landsat } 5 \\
\text { ETM }\end{array}$ & LEDAPS & ee.ImageCollection("LANDSAT/LT05/C01/T1_ANNUAL_BAI \\
\hline $2013-2019$ & $\begin{array}{c}\text { Landsat } 8 \\
\text { OLI }\end{array}$ & LaSRC & ee.ImageCollection("LANDSAT/LC08/C01/T1_ANNUAL_BAI \\
\hline
\end{tabular}


que ocurrió el incendio. Finalmente, estos vectores se sobrepusieron a los datos de IAQ para verificar el nivel de ajuste entre los dos conjuntos de datos.

\section{Preprocesamiento y análisis espacial}

Una vez recopilados todos los polígonos de incendio para el periodo de estudio, fueron incorporados en el programa QGIS (v. 3.2.2) [16], para depurar y agrupar los incendios según su recurrencia. La depuración consistió en corregir todos los errores topológicos contenidos en los vectores, como por ejemplo polígonos sin cierre, sobreposición de vértices y datos sin atributos. Los datos de IAQ fueron categorizados de acuerdo con la recurrencia de incendios en: 0 incendios, 1 incendio, de 2 a 3 incendios, y más de 4 incendios utilizando la herramienta Map Algebra de QGIS.

Para construir el conjunto de datos necesarios para realizar el análisis estadístico, el área de estudio se dividió en 2280 hexágonos de 25 hectáreas que fueron utilizados como unidades mínimas de mapeo y muestra, siguiendo el método de Guzmán y Vega [17]. Sin embargo, debido a que no todos los hexágonos abarcaron 25 hectáreas, como resultado de los límites con el océano que rodea el área de estudio, a la hora de seleccionar las muestras sólo se utilizaron hexágonos mayores de 6.25 hectáreas del área. Para cada año los datos de IAQ fueron extraídos utilizando como referencia los 2280 hexágonos en el programa QGIS (v. 3.2.2) [16], por medio de la extensión Zonal statistics, que permite calcular varios valores (suma, promedio, mediana, desviación estándar, etc.) de los píxeles de una capa rasterizada con la ayuda de una capa vectorial poligonal.

Con esta herramienta fue posible conocer para cada uno de los 2280 hexágonos, el valor promedio y desviación estándar de los datos de IAQ por año de los píxeles cubiertos por el hexágono. Posteriormente, para cada categoría de recurrencia de incendios ( 0 incendios, 1 incendio, de 2 a 3 incendios, y más de 4 incendios) fueron seleccionados 25 hexágonos por año (Con excepción del año 2012, ya que, no fue posible adquirir datos para este año), recolectando en cada año los mismos 25 hexágonos de cada grupo de recurrencia, para un total de 2200 muestras (Figura 2).

\section{Análisis estadístico}

Los análisis estadísticos para evaluar las diferencias en los valores de IAQ fueron realizados en el entorno y lenguaje de programación $R$ (v.3.4.4) [18], utilizando el

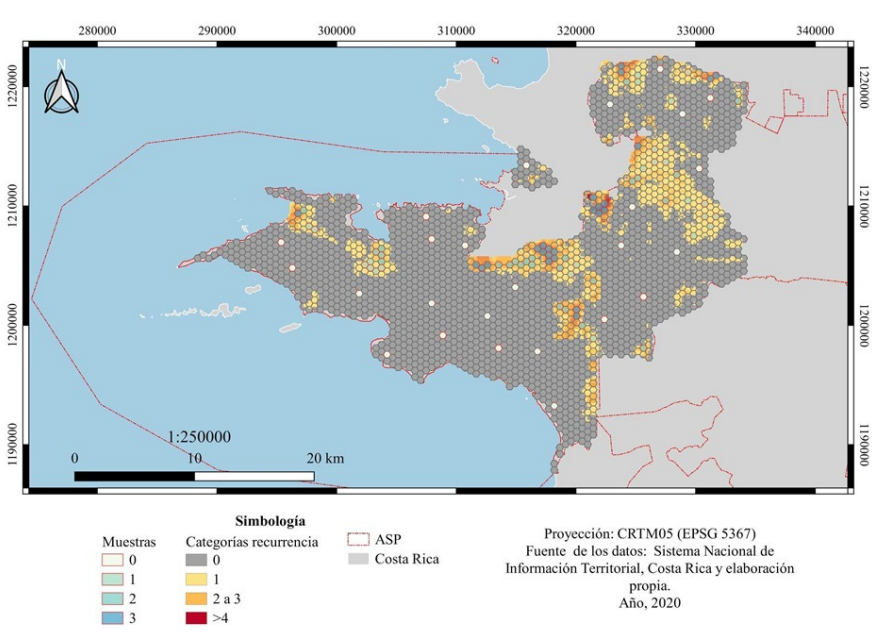

Figura 2. Muestreo de categorías de recurrencia de incendios en la región de bosque seco tropical del ACG, Costa Rica 1997 al 2019.

Figure 2. Sampling of fire recurrence categories in the ACG tropical dry forest region, Costa Rica 1997 to 2019

valor del promedio de IAQ para cada hexágono, el año de colecta y el grupo de recurrencia. Para conocer si los datos poseen una distribución normal o no, y así definir el tipo de prueba estadística a utilizar para analizar los datos, se aplicó una prueba de normalidad ShapiroWilk, que identifica si la distribución de los datos es igual o diferente a una distribución normal o gaussiana. Posteriormente, debido a la distribución no normal de los datos fue necesario realizar dos transformaciones. La primera fue transformar a factor las categorías de recurrencia de incendios, utilizando el comando as.factor de la librería dplyr. La segunda transformación necesaria fue aplicar una transformación de rangos al valor promedio de IAQ, utilizando el comando rank de $\mathrm{R}$. Luego se implementó un modelo lineal de efectos mixtos (LME, por sus siglas en inglés), por medio de la función Imn de la librería nIme utilizando la ecuación 2.

lme $($ fixed $=$ rmean $\sim$ cat, random $\sim 1 \mid$ year $)$

Donde Ime representa la función; fixed un objeto de fórmula lineal de dos lados que describe la parte de efectos fijos del modelo, con la respuesta a la izquierda de un operador $\sim$ y los términos, separados por operadores; rmean indica el valor promedio de IAQ para cada hexágono; random $=\sim 1$ especifica la ocurrencia de efectos aleatorios; y lyear especifica que las medidas se repiten para cada año. 
Finalmente, para conocer si existen diferencias en los valores de IAQ en el tiempo según las categorías de recurrencia de incendios forestales, a los resultados del modelo generado se les aplicó un análisis de varianza (ANDEVA) con medidas repetidas al modelo generado, utilizando la función anova de R. Asimismo a los resultados del ANOVA se les aplicó una prueba de Comparación múltiple de medias por contraste de Tukey, utilizando la librería TukeyHSD de R.
Cuadro 2. Resultados del análisis estadístico de las cuatro categorías de recurrencia de incendios forestales en el ACG, Costa Rica 1997 al 2019.

Table 2. Results of the statistical analysis of the four categories of recurrence of forest fires in the ACG, Costa Rica 1997 to 2019.

\begin{tabular}{|cccccc|}
\hline & ANDEVA-LME & & \multicolumn{3}{c|}{$\begin{array}{c}\text { Prueba de } \\
\text { normalidad } \\
\text { Shapiro-Wilk }\end{array}$} \\
\hline & GL & F-value & p-value & W & p-value \\
\hline Intersección & 1 & 223.271 & $<.0001$ & 0.94601 & 0.00111 \\
\hline Categoría & 3 & 9.078 & $<.0001$ & & \\
\hline
\end{tabular}

\section{Resultados}

La distribución de los valores de IAQ para el período 1997 al 2019 presentan una distribución significativamente diferente a la de una distribución normal $(\mathrm{SW}=0.94601$, $p$-value $=0.00111)$. Se da una agrupación de valores en las primeras categorías de recurrencia, es decir, en el ACG la mayoría de los incendios ocurre una o varias veces en un sitio. Los resultados del ANDEVA del modelo lineal de efectos mixtos generados, evidenciaron que existen diferencias significativas en el promedio de los valores de IAQ para categorías de recurrencia de incendios forestales (Cuadro 2).

En general, en todos los años el IAQ mostró diferencias en los valores para las cuatro categorías según la recurrencia de incendios forestales (Figura 3). Con algunas excepciones como los años 2004, 2006, 2008 y 2016, el IAQ de las zonas recurrentemente quemadas (más de 4 incendios), presentan los valores más altos. Similar es el comportamiento de las zonas que han sufrido 2 o 3 incendios, sin embargo, la tendencia es menos evidente. Por otro lado, las zonas que solo han sido afectadas por un único incendio o no han sido afectadas del todo, muestran valores de IAQ menores que las zonas quemadas de forma recurrente.

El análisis de comparación múltiple de medias por contraste de Tukey aplicado a los resultados del ANDEVA mostró diferencias significativas entre las medias de dos grupos de recurrencia de incendios $(p<.05)$. Un primer grupo conformado por las categorías de 0 incendios y 1 incendio y un segundo grupo conformado por las categorías de 2 a 3 incendios y más de 4 incendios.

\section{Discusión}

Los análisis post incendio como el IAQ permiten identificar los cambios inducidos por el fuego en la respuesta espectral de las coberturas de la tierra, ya sea por cambios estacionales de la vegetación o alteraciones

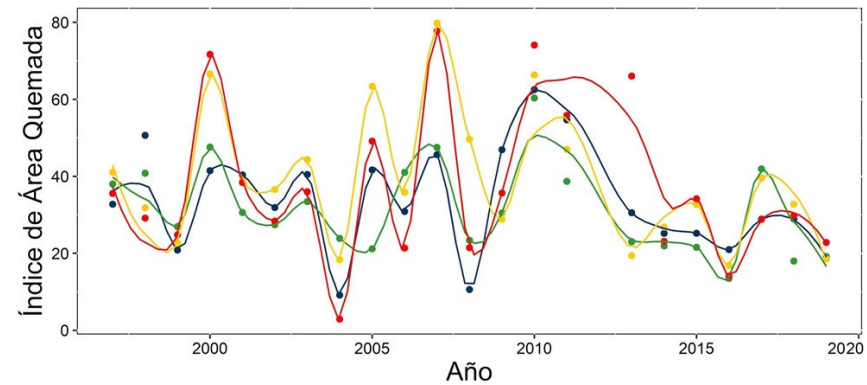

Figura 3. Valores anuales de IAQ de las cuatro categorías de recurrencia de incendios forestales en el Área de Conservación Guanacaste, Costa Rica, 1997 al 2019.

Figure 3. Annual BAl values of the four categories of forest fire recurrence in the Guanacaste Conservation Area, Costa Rica, 1997 to 2019 .

antropogénicas [19]. La utilidad del índice IAQ en incendios forestales se debe a que es más sensitivo a las áreas quemadas que otros índices como el NDVI, GEMI o SAVI, debido a que, se diseñó para enfatizar el carbón en imágenes post fuego [8]. Sin embargo, con coberturas de baja reflectancia como los cuerpos de agua o las sombras de nubes, el IAQ presenta algún potencial de confusión [8]. Asimismo, un aspecto discutible es que el IAQ fue desarrollado en ambientes mediterráneos; sin embargo, el IAQ puede ser aplicable a otros biomas como los bosques tropicales secos, dependiendo de la perdurabilidad del carbón después del fuego, lo cual puede ser desde meses como en las regiones boreales, semanas o días como en los trópicos [10].

En el ACG, la tendencia en los valores del IAQ entre el período de 1997 al 2019, evidenció que las áreas quemadas que han sido afectadas por un único incendio forestal no muestran diferencias con respecto a los valores obtenidos por el índice IAQ en áreas donde 
nunca ha ocurrido un incendio. Lo anterior podría indicar que estas áreas recuperan sus propiedades después de ser afectadas por un único incendio. Por lo general, las áreas identificadas en el ACG, con menor recurrencia, pero que sí han sufrido al menos un único incendio, experimentan una rápida colonización de especies pioneras como arbustos en el primer año, para luego mediante el rebrote y dispersión de semillas incrementar la cobertura vegetal en los años posteriores. Wittenberg et al. [20], reportaron un comportamiento similar en áreas que fueron afectadas por un incendio, donde el crecimiento de la vegetación y el aumento de la riqueza de especies fue significativo durante los primeros tres años después de ocurrido el incendio.

Por otro lado, en áreas quemadas donde han ocurrido de 2 a 3 y más de 4 incendios, no se observaron diferencias en cuanto a la tendencia en los valores del índice IAQ, lo que podría indicar que la recuperación en esas áreas no es tan rápida o evidente como en las áreas donde solo ha ocurrido un solo incendio. Por consiguiente, esto podría estar derivando a un proceso de degradación, dado que, la recuperación de los bosques después de las perturbaciones puede verse limitada por múltiples mecanismos [21].

En el ACG, las áreas donde hay una recurrencia de incendios año tras año, se presenta un escenario de colonización por plantas invasoras no forestales, lo que podría estar llevando estadios de sucesión secundaria a estadios equivalentes a pastizales o bosques jóvenes, esto debido a la disminución de las fuentes locales de semillas tras los incendios. Costa et al. [22], mencionan que la composición por especies de los bosques en regeneración es condicionada por la capacidad de las especies de tolerar el fuego, debido a que, el establecimiento de nuevos individuos puede verse limitado por la disponibilidad de semillas, la capacidad de germinación de semillas y la capacidad de supervivencia de estas.

La recuperación de los bosques después de un incendio forestal es un proceso lento, por ejemplo, según Oliveira et al. [23] se toma al menos 45 años para que un bosque secundario estacional obtenga valores de biomasa cercanos a los de bosques nativos luego de un incendio. Así mismo, Cárdenas-Salgado y Pizano [24], mencionan que la regeneración a partir de semillas es cada vez más difícil debido al incremento de las presiones antropogénicas como los incendios forestales sobre los ecosistemas de bosque seco tropicales.

Lo anterior podría explicar la significativa diferencia en los valores de IAQ entre áreas sin incendios o al menos un incendio y áreas con más de dos incendios, ya que, en los bosques secos tropicales los procesos de recuperación luego de un disturbio como un incendio forestal, pueden ser altos; sin embargo, dependen de las fuentes de semillas para la supervivencia y el rebrote de los árboles [22]. En la Amazonia, Flores et al. [25], encontraron que dos incendios pueden socavar la capacidad de recuperación de los bosques, debido a que, después de un segundo incendio, persiste un estado no forestal; y por lo que, los pocos árboles reclutados aparentemente no logran suprimir las plantas herbáceas [24]. Por lo tanto, los altos valores de IAQ en el ACG en áreas con más de dos incendios podrían estar asociados a cambios en el banco de semillas que se deriva en reclutamiento escaso de especies forestales y una alta dominancia de plantas herbáceas.

Aunque el fuego en muchas ocasiones está ligado al mantenimiento de terrenos para servicios tangibles como la ganadería o agricultura, este afecta de forma directa la biodiversidad y los ecosistemas forestales propiciando a largo plazo una degradación de los servicios ecosistémicos de los bosques. Por ejemplo, en los bosques tropicales, los incendios forestales reducen la capacidad de evapotranspiración y retención de agua en las copas de los árboles y suelos [25], [26]. Además, la recurrencia y severidad de los incendios forestales pueden exacerbar la pérdida de servicios ecosistémicos como la calidad de agua, contaminación del aire, almacenamiento de carbono, composición y estructura de la biodiversidad [23], [26], [27].

\section{Concluciones}

Este estudio demostró que las áreas recurrentemente quemadas por incendios forestales muestran un comportamiento diferenciado en el IAQ a lo largo de todos los años. Se encontró que, a mayor recurrencia de incendios, mayores serán los valores de IAQ, mientras que las áreas con menos incendios mostraron valores bajos de IAQ. Lo anterior podría ser consecuencia de un cambio en la composición de especies, debido al surgimiento de especies pioneras. Además, los fuertes procesos de degradación en la cobertura y el suelo posterior a un incendio forestal inhiben la proliferación de más especies debido a las pocas fuentes de bancos de semillas en la zona afectada.

Los valores de IAQ mostrados en este trabajo responden a los valores de áreas quemadas en la región del bosque seco tropical del Área de Conservación Guanacaste, Costa Rica, por lo tanto, se considera que los resultados podrían ser replicados a lo largo del Corredor Seco Mesoamericano con el fin de comparar similitudes y crear sistemas de alerta y monitoreo a partir del uso del $I A Q$ en zonas donde se presenten incendios forestales de forma recurrente y así profundizar en el conocimiento 
sobre la severidad de los incendios y los procesos de recuperación de los bosques.

\section{Agradecimientos}

Al personal del Área de Conservación de Guanacaste por su apoyo en el trabajo de campo. A Julio Díaz y Didi Guadamuz del Programa de Manejo del Fuego del ACG, por los datos de polígonos de áreas quemadas y todo el apoyo logístico brindado. A Adolfo Quesada-Román por su colaboración en la revisión preliminar del documento. A la Vicerrectoría de Investigación de la Universidad Estatal a Distancia (UNED), Costa Rica por el apoyo financiero.

\section{Referencias}

[1] M. A. Cochrane et al., "Positive feedbacks in the fire dynamic of closed canopy tropical forests," Science (80). vol. 284, no. 5421, pp. 1832-1835, Jun. 1999.

[2] T. Devisscher, Y. Malhi, V. D. Rojas Landívar, and I. Oliveras, "Understanding ecological transitions under recurrent wildfire: A case study in the seasonally dry tropical forests of the Chiquitania, Bolivia," For. Ecol. Manage., vol. 360, pp. 273-286, 2016.

[3] S. Otterstrom, M. Schwartz, and I. Velázquez-Rocha, "Responses to Fire in Selected Tropical Dry Forest Trees," Biotropica, vol. 38, no. 5, pp. 592-598, 2006.

[4] D. C. Morton, Y. Le Page, R. DeFries, G. J. Collatz, and G. C. Hurtt, "Understorey fire frequency and the fate of burned forests in southern Amazonia.," Philos. Trans. R. Soc. Lond. B. Biol. Sci., vol. 368, no. 1619, p. 20120163 , 2013.

[5] M. A. Cochrane and M. D. Schulze, "Fire as a recurrent event in tropical forests of the eastern Amazon: effects on forest structure, biomass, and species composition," Biotropica, vol. 31, no. 1, pp. 2-16, 1999.

[6] D. K. Bolton, N. C. Coops, and M. A. Wulder, "Characterizing residual structure and forest recovery following highseverity fire in the western boreal of Canada using Landsat time-series and airborne lidar data," Remote Sens. Environ., vol. 163, pp. 48-60, 2015.

[7] E. Uuemaa, Ü. Mander, and R. Marja, "Trends in the use of landscape spatial metrics as landscape indicators: A review," Ecol. Indic, vol. 28, pp. 100-106, 2013.

[8] E. Chuvieco, M. P. Martín, and A. Palacios, "Assessment of different spectral indices in the red-near-infrared spectral domain for burned land discrimination," Int. J. Remote Sens, vol. 23, no. 23, pp. 5103-5110, Jan. 2002.

[9] M. M. Verstraete and B. Pinty, "Designing optimal spectral indexes for remote sensing applications," IEEE Trans. Geosci. Remote Sens., vol. 34, no. 5, pp. 1254-1265, 1996.

[10] E. Chuvieco et al., "Historical background and current developments for mapping burned area from satellite Earth observation," Remote Sens. Environ., vol. 225, no. November 2018, pp. 45-64, 2019.

[11] D. Vargas-Sanabria and C. Campos-Vargas, "Modelo de vulnerabilidad ante incendios forestales para el Área de Conservación Guanacaste, Costa Rica," UNED Res. J., vol. 10, no. 2, pp. 435-446, Sep. 2018.

[12] D. Vargas-Sanabria and C. Campos-Vargas, "Sistema multi-algoritmo para la clasificación de coberturas de la tierra en el bosque seco tropical del Área de Conservación Guanacaste, Costa Rica," Rev. Tecnol. en Marcha, vol. 31, no. 1, p. 58, 2018.

[13] G. A. Sánchez-Azofeifa et al., "Can terrestrial laser scanners (TLSs) and hemispherical photographs predict tropical dry forest succession with liana abundance?," Biogeosciences, vol. 14, no. 4, pp. 977-988, 2017.

[14] J. P. Arroyo-Mora, G. A. Sanchez-Azofeifa, M. E. R. Kalacska, B. Rivard, J. C. Calvo-Alvarado, and D. H. Janzen, "Secondary Forest Detection in a Neotropical Dry Forest Landscape Using Landsat 7 ETM+ and IKONOS Imagery1," Biotropica, vol. 37, no. 4, pp. 497-507, Dec. 2005.

[15] N. Gorelick, M. Hancher, M. Dixon, S. Ilyushchenko, D. Thau, and R. Moore, (2017). Google Earth Engine: Planetaryscale geospatial analysis for everyone. Remote Sensing of Environment. vol. 202, no. 1, pp. 18-27, Dec. 2017.

[16] QGIS Development Team. QGIS Geographic Information System. Open Source Geospatial Foundation. 2019. [Online] Disponible: http://qgis.org. [Accessed Mar. 11, 2019]

[17] J. Guzmán and H. Vega, "Is forest cover conserved and restored by protected areas?: The case of two wild protected areas in the Central Pacific of Costa Rica," Int. J. Trop. Biol. Conserv., vol. 63, no. 3, pp. 579-590, 2015.

[18] [18] R Core Team. R: A language and environment for statistical computing. R Foundation for Statistical Computing, Vienna, Austria. 2014. [Online] Disponible: http://www.R-project.org/. [Accessed Jun. 18, 2019 ]

[19] L. Giglio, I. Csiszar, and C. O. Justice, "Global distribution and seasonality of active fires as observed with the Terra and Aqua Moderate Resolution Imaging Spectroradiometer (MODIS) sensors," J. Geophys. Res. Biogeosciences, vol. 111, no. G2, Jun. 2006.

[20] L. Wittenberg, D. Malkinson, O. Beeri, A.Halutzy, and N. Tesler, "Spatial and temporal patterns of vegetation recovery following sequences of forest fires in a Mediterranean landscape, Mt Carmel Israel", Catena, vol. 71, no. 1, pp. 76-83, Sep. 2007.

[21] R. L. Chazdon, "Tropical forest recovery: legacies of human impact and natural disturbances," Perspect. Plant Ecol. Evol. Syst., vol. 6, no. 1-2, pp. 51-71, Jan. 2003.

[22] M. B. Costa, L. F. T. De Menezes, and M. T. Nascimento, 
"Post-fire regeneration in seasonally dry tropical forest fragments in southeastern Brazil," An. Acad. Bras. Cienc. vol. 89, no. 4, pp. 2687-2695, 2017.

[23] A. T. Oliveira Filho, D. A. Carvalho, E. A. Vilela, N. Curi, and M. A. L. Fontes, "Diversity and structure of the tree community of a fragment of tropical secondary forest of the brazilian Atlantic Forest domain 15 and 40 years after logging," Rev. Bras. Botânica, vol. 27, no. 4, pp. 685-701, Oct. 2004.

[24] C. Pizano, and J. C. Cárdenas-Salgado, "Efecto de temperaturas que simulan incendios sobre la germinación de semillas de un bosque seco tropical," Colomb. For., vol. 22, no. 2, pp. 55-66, Jun. 2019.

[25] B. M. Flores, R. Fagoaga, B. W. Nelson, and M. Holmgren, "Repeated fires trap Amazonian blackwater floodplains in an open vegetation state," J. Appl. Ecol., vol. 53, no. 5, pp. 1597-1603, Oct. 2016.

[26] J. Schmerbeck and P. Fiener, "Wildfires, Ecosystem Services, and Biodiversity in Tropical Dry Forest in India," Environ. Manage., vol. 56, no. 2, pp. 355-372, 2015.

[27] C. Lee, C. Schlemme, J. Murray, and R. Unsworth, "The cost of climate change: Ecosystem services and wildland fires," Ecol. Econ., vol. 116, pp. 261-269, Aug. 2015. 